\title{
A review on association and correlation of genetic variants with eating disorders and obesity
}

\author{
Sayed Koushik Ahamed ${ }^{1}$, Md Abdul Barek², Uthpall Kumar Roy ${ }^{1}$, Md Kouser ${ }^{1}$, Md Sharif Reza², \\ Afifa Binthe Mannan ${ }^{3}$, Md Ashraful Alam ${ }^{4}$ and S. M. Naim Uddin ${ }^{4^{*}}$ (D)
}

\begin{abstract}
Background: Now, eating disorders and obesity and their correlations are danger signal in worldwide which is caused by multifactor and associated with significant mortality and morbidity.

Main body: Every aspect of a patient's life is influenced by eating disorders and obesity and their correlations. Due to frequent seeing of obese patients, eating disorders have been included in the review as they can sometimes be associated with obesity. However, it should be noted that most patients having eating disorder are at risk to be obese or overweight. This research explores the risk factors for the two disorders, as well as the assessment of medical complications and treatment recommendations for the disorders. In these two disorders, there is also a correlation. The essential consideration is that eating disorders are impulse-control disorders which are similar to addictive behaviors in some aspects. So it is a crying need to treat a patient with obesity and eating disorders simultaneously to ensure success. Genome-wide association studies (GWASs) have increased our knowledge of the pathophysiology of eating disorders (EDs) and obesity and their correlation.
\end{abstract}

Conclusion: This review enlightens on the summary of eating disorder, obesity, genotypic traits, molecular relations, interaction, correlation, and effect of eating disorder and obesity which outline potential future directions and clinical implications for patients with EDs and obesity.

Keywords: Eating disorders, Obesity, Genome, traits

\section{Background}

Eating disorders can be categorized into a class of mental illnesses that could result in numerous somatic and psychiatric complications and have penchant to perturb living quality as well as mortality in some cases. This psychiatric condition has increasingly been able to seek attention among world health fraternities over the past 20 years [1]. In accordance with the National Eating Disorder Association, not less than 70 million people across the world including both the male and female have been reported to experience eating disorders during their

\footnotetext{
* Correspondence: pharma.naim@cu.ac.bd

${ }^{4}$ Department of Pharmacy, University of Chittagong, Chittagong 4331, Bangladesh

Full list of author information is available at the end of the article
}

lifetime [2]. In every $62 \mathrm{~min}$, the earth witnesses at least one of its inhabitants being deceased directly due to this nuisance [3]. The outbreak of these psychological disorder is on the rise in numerous countries and it was particularly predominant in females with young age [4], in Western and affluent countries [5] during the late twentieth century presumably by the virtue of factors related to economy and culture. Albeit globally, the ranking had been quite steady during the time period of 1990 to 2013 ( shifting from 13th position in 1990 to 12th position in 2013); ranking of middle and lowincome countries grew from 58th position in 1990 to 46th position in 2013 [6]. In recent time, eating disorders have been widely burgeoning among people dwelling in non-Western countries, mainly South Asia, China, 
and Middle East [7]. At present, worldwide pervasiveness of eating disorders is prominent, and hence Asia is also encountering overwhelming health burdens, though sparseness in terms of epidemiological information exists in Pacific Island and Asian countries [8]. The surges of EDs over the last 30 years have enlightened the intricate intertwining between pathology and culture. The propagation of EDs across various part of Asian region has combined with the time of unprecedented development and widespread economic and social revolution throughout much of the arena [9]. In Asia proper, only a few cases of EDs were recorded in certain countries before the 1990s, such as Singapore, India, Malaysia and among Chinese staying in Hong Kong (once under British control), but as a whole, EDs had been extensively absent in Asia except for Japan where EDs were reported to come into view in the mid-1970s [10]. Reportedly, by the end of the twentieth century, disorders in the eating behaviors and attitude enhanced considerably across Asian high-income young females, whereas clinical EDs crossed the boundaries of Japan to Hong Kong, Singapore, Taiwan, and Korea [11]. It is stated that EDs have proliferated among Asian population through the supposedly "Asian miracle" of economic revolution as it stretches across the territory [10]. Initially, EDs were found less prevalent in low-developed and relatively indigent Asian countries such as Myanmar, Thailand, India, Indonesia, Bangladesh, Philippines, Cambodia, China, Laos, and Vietnam [11]. However, evidence points out that there was a sharp increase in the cases of EDs across the territory varying explicitly with economic development and concomitant urbanization and industrialization. Obesity is one of the risk factors for the commencement of eating disorders as it is linked with high degree of body image discontentment and dieting pattern, and can certainly be also viewed as a tag of disordered eating. Accordingly, obesity, a comprehensive event of the twenty-first century is a pivotal causative factor for a lot of other metabolic disorders. WHO says, in 2014 over 1.9 billion people of 18 years old and above were found to be overweight; of these, more than 600 million people were obese, whereas about 15\% women deemed obese across the globe [12]. In general, around the world, obesity and overweight kill more number of people in comparison with underweight. In 2016, 41 million children aged below 5 were obese or overweight while at the same year more than 340 million adolescents and children aged 5-19 were estimated to be obese or overweight [13]. Eating disorders and obesity are usually considered as two separate problems and are tackled by multidisciplinary professionals via the employment of distinctive clinical approaches and theoretical models. However, the current WHO standard data, garnered from a total of 79 developing countries along with some industrialized ones, reveals that the number of overweight children in the world are 110 million [14]. In several developing countries, the increasing number of overweight and obese children and adolescents has been well reported. Insufficiency in macro- and micronutrients has long been a major issue for children in low-income countries. Given a persistently high burden of malnutrition, Indo-Asian countries are now confronted with the peculiar challenge of a rapid increase in childhood obesity [15]. Over the last few decennaries, majority of Asian countries have noticed a drastic surge in the number of obese and overweight people, with the figures being soared up to many times and the degree of extension differs based on the diversification of various countries. One of the health surveys carried out in Bangladesh asserted that the presence of obesity and overweight (11.86\% vs. $14.86 \%)$ were markedly greater in males (1.29-fold) in comparison to females. Unlike the average waist-circumference in male was prominently higher than in females, the ratio of waist to height (WHtR) was seen greater in females when correlated with males [16]. Teenaged girls have a potentiality to endure more than single disorder or to move from one disease to another with varied levels of severity $[17,18]$. Conversely, a meta-analysis divulged that, pooled prevalence rate for obesity and overweight during the year of 2000 and 2015 among the adolescents and children was 9.7\% and $7.9 \%$ respectively [19] and around $37.6 \%$ university going pupils in Bangladesh were deemed to be predisposed to eating disorder [20]. A variety of psychological hypotheses are available on eating disorders and out of them a hypothesis titled as "thrifty genotype" proposes that binge eating is sort of a psychological habituation that helps avoid malnutrition, regulated reproduction, and survival amid famines. Whereas, another hypothesis popularly known as "intra-sexual competition" has come up with the concept that the extreme intra-sexual contest for mates is the eventual reason for eating disorders. This hypothesis figures out that the shape of a woman's body is a marker of her reproductive potential, reproductive history, as well as mate value, partially signaled by BMI or body mass index and waistto-hip ratio. It can be acknowledged that International conferences attended by the obesity researchers and specialists and arranged by the national and international associations of obesity often have dearth of contents on eating disorders. Notwithstanding that they have own community and professional group to eating disorder experts, they hardly manage to attract a great deal of commercial sponsorships in order to hold their meetings. This aberration is largely mirrored in varying public media perception to the disorders-blame or reluctance in obesity cases, and pity and awe concerning eating disorders. Antipathy between these two is generally 
buttressed by the media through its overly simplified portrayal of scientific attainments and its extreme obsession with celebrity and body image. Women are often derided alternately for losing or gaining weight under the media limelight, with accompanying remarks which either rebuke them for their excessive self-indulgence or applaud them on their resiliently obtained thinness. Therefore, it has become an utmost priority to determine the long-standing characteristics of the both clinical problems of eating disorder and obesity.

Table 1 Classification, causes, sign and symptoms, treatment of eating disorders

\begin{tabular}{lll}
\hline Classification & Causes & Sign and symptoms \\
\hline Anorexia & Anorexia nervosa is linked to biological, & marked weight loss, vomiting, chronicity, \\
nervosa & cultural and social factors, family, parenting & cardiac muscle wasting, psychiatric \\
& style, environmental influences, parental & comorbidity, hypoglycemia, psychosocial \\
& discord, and household stress. & problem, passive-aggressive and obsessive \\
& & compulsive disorder, heart failure, depres- \\
& sion, hypoproteinuria, extended corrected \\
& QT interval, mineral and vitamin inad- \\
& equacy, electrolyte disorder are common \\
& [23].
\end{tabular}

Treatment

- Medication: (SSRI or selective serotonin reuptake inhibitors and antidepressants) [24], TCA or tricyclic antidepressant drugs $[25,26]$, antipsychotics [27], hormones [28], antiepileptics [29], nutritional supplement [30], weight restoration, and nutritional intervention.

- Psychosocial interventions (CBT or cognitive behavioral therapy [31], CAT or cognitive analytic therapy [32])

- Family therapy [33]

- Psychosocial interventions [34]

Bulimia Mood disorder, physical and sexual abuse, nervosa obesity, parental obesity, low self-esteem, substance abuse, disturbed family dynamics, perfectionism, early menstruation in eating disorder patients, and parental shape/weight concern [35].
Bulimia nervosa has been observed to manifest binge eating symptoms, depression and anxiety, compensatory behavior, higher rates of obesity, sexual and physical abuse, mood disorder, perfectionism, low self-esteem, early menartal weight/shape concern [36]. che, disturbed family dynamics, and paren-

- Medications: (SSRI, antidepressants, trazodone, 5- $\mathrm{HT}_{3}$ antagonist, MAO inhibitors) [35].

- Psychosocial interventions [37] (dialectic behavior therapy, CBT).

- Self-help trials, family therapy, 12-step programs/support group [38].

- Psychosocial interventions plus medication $[38,39]$ (CBT and tricyclic antidepressant, CBT and SSRI, CBT and multiple drugs).

- Additional interventions (light therapy, guided imagery therapy, crisis prevention [37]).

- Medications [43] (SSRI, anti-obesity, anticonvulsants)

Binge eating is referred as the majo symptom of BED; but, every individual who binge eats does not possess BED. A person can binge eat without acknowledging number of negative psychological, social or physical consequences of BED. The aforementioned example can, at the most, be regarded as eating problem except for a disorder. It is somewhat problematic to provide a precise definition of binge eating, however episodes of binge eating in BED usually have the following features [41, 42]:

- Eating much quickly than the usual, may be within short time space.

- Eating up to the time of perceiving uncomfortably full.

- Eating a huge volume even when do not have appetite for food.

- Losing control over what or how much is eaten

- Binges could be premeditated associating with buying certain binge foods, allocation of particular time for binging, occasionally at night.

- Eating confidentially or alone due to the feeling of discomfort over the volume of food taken.

- There could be bewildered psychological state at the time of binge.

- Being unable to recall what was consumed following the binge

- Sense of guilt, disgust or shame after a food binge.
- Multidisciplinary sequential strategy [43] (Psychoeducational and behavioral interventions)

- Psychotherapies [40] (DBT or dialecticbehavioral therapy, CBT)

- Personal motivation and counseling

- Bariatric surgery.

- Administration of numerous SSRIs, e.g. sertraline, citalopram, fluvoxamine and fluoxetine, etc. [44].

- Other treatments available for BED are lifestyle interventions such as peer support groups, hormonal abnormalities investigation and weight training. Concentrating on optimistic mindset and self-love is effective for many [45]. 


\section{Main text}

\section{Eating disorder and obesity}

What does obesity and eating disorder mean?

Obesity is a long-term clinical condition which evokes plethora of other comorbid diseases and is accountable for medical morbidity to a great extent. With the global burst of corpulence, consciousness and research regarding obesity has heightened which ultimately leads to the ground breaking innovation of noble drugs, surgeries, and diets. Having stated that, yet the problem has kept hiking up at a substantial rate, particularly in adolescents. Eating disorders refer to illnesses that can be characterized by the presence of severe anguish or anxiety about one's own body mass or shape and undisciplined eating habits. Eating disruptions may involve insufficient or excessive consumption of food than the usual which is likely to inflict damage to individual's weal. Anorexia nervosa, binge eating disorder, and bulimia nervosa are the three most prevalent eating disorders. Anorexia nervosa, commonly termed as simple anorexia, is explicated as a type of eating disorder diagnosed by the presence of diminished body weight, fear of being chubby, dietary restriction, and an intense craving to become skinny [21]. Total death tolls caused by anorexia nervosa is the highest among all the psychiatric disorders [22] and about $0.3 \%$ young women have been found to be affected by it. Bulimia nervosa is a condition where someone becomes exceedingly preoccupied with his/her body shape and weight, with regular incidents of unrestrained gluttony accompanied by measures to subdue the appalled effects of eating in excess. Finally, binge eating disorder can be construed as a sort of severe eating disease characterized by frequent intake of abnormally large volume of diet and feeling of being incapable to put an end to eating. Treatment for eating disorders should necessarily be personalized on the basis of symptom severity (Table 1).
The relationship between obesity and eating disorders

Until the inclusion of BED (binge eating disorder) and compensatory behaviors of obesity, there had been conduction of extensive studies in the field of compulsion-induced over-eating and binge eating on clinical platform for some period of 50 years. In the decade of the 1990s, BED was formally subsumed in the categorization of diseases, and as a matter of fact even now researchers have an inclination to carry out studies on it compared to diagnostic status (Fig. 1). Recurrent self-indulgent overeating episodes characterize BED, with omitting the abuse of laxative or compensatory vomiting which actually represent bulimia nervosa. Despite majority of obese persons are not presented with BED, susceptibility to BED continues to rise with augmenting obesity. BED exists more commonly in females than the male counterparts. Surveys have managed to shed light upon the verity that, around $1-2 \%$ people expose to BED at some stages of their lifespan, with about $0.1-1 \%$ people being afflicted in a certain year. Among subjects who are seeking treatment for weight loss have been observed to show varied rates of $1.3-30 \%$ [46]. BED conceptualizations debate that it can be distinctly distinguished from anorexia; however, it is in no way a useful subclass of obesity [47]. Moreover, there has been a suggestion that treatments concentrated on BED could be succeeded but have a meager effect on the loss of individual's weight. Weight-loss treatments in obese people are effective in an equal manner (modestly) with the presence or absence of BED, but patients with a mood disorder (also referred as mood affective or affective disorder and is more common in BED) do not improve much. Gain speculation is a term which means abnormal dietary intake, Unhealthy diet, lifestyle, certain medication, and other medical problems. It is thought to be of a risk factor in terms of weight and BED might impart contribution to the escalation in obesity [48].

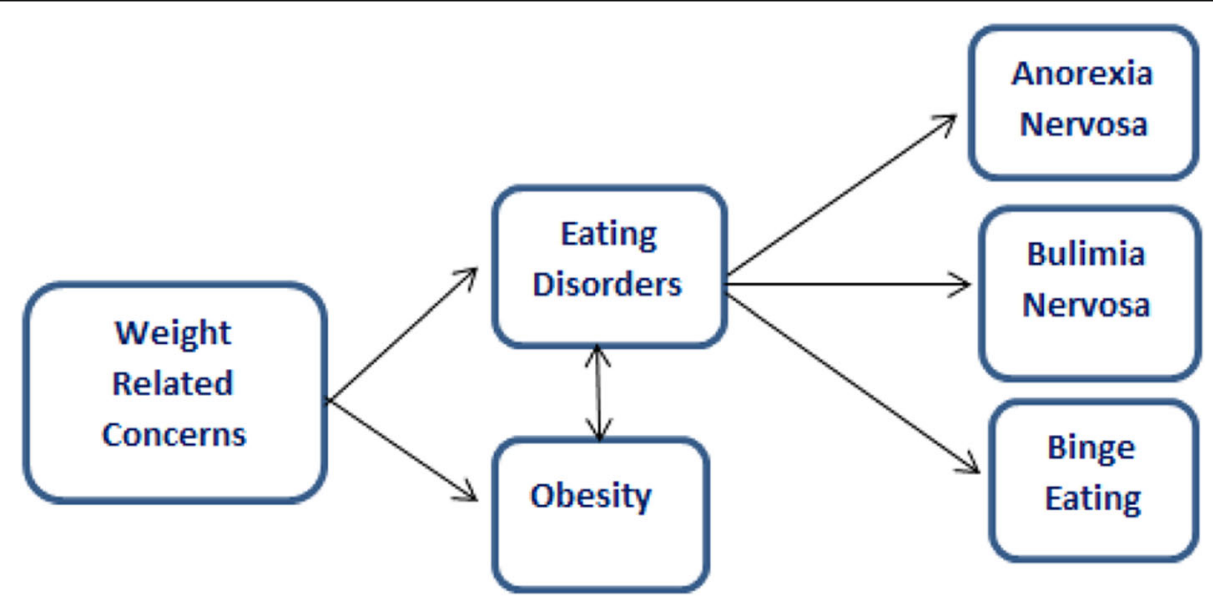

Fig. 1 Obesity and eating disorders and their co-relationships 
Table 2 Overview of candidate gene findings in eating disorders

\begin{tabular}{|c|c|c|c|}
\hline Gene & Description & rs Number & References \\
\hline \multirow[t]{2}{*}{ HTR1D } & Serotonin receptor $1 \mathrm{D}$ & rs6300 & [51] \\
\hline & & $\begin{array}{l}\text { rs674386 } \\
\text { rs } 856510\end{array}$ & [52] \\
\hline HTR2A & Serotonin receptor $2 \mathrm{~A}$ & rs6311 & {$[53-56]$} \\
\hline HTR2C & Serotonin receptor $2 \mathrm{C}$ & rs6318 & [57] \\
\hline \multirow[t]{4}{*}{ DRD2 } & Dopamine receptor D2 & rs1799732 & {$[58-60]$} \\
\hline & & rs6277 & {$[59,60]$} \\
\hline & & rs6278 & [58] \\
\hline & & rs2283265 & [60] \\
\hline DRD3 & Dopamine receptor D3 & rs6280 & [61] \\
\hline DRD4 & Dopamine receptor D4 & rs1800955 & [62] \\
\hline COMT & Catechol-O-methyltransferase & rs4680 & {$[63,64]$} \\
\hline ANKK1 & Ankyrin repeat and kinase domain containing 1 & rs1800497 & {$[59,60]$} \\
\hline LEP & Leptin & $\begin{array}{l}\text { rs13228377 } \\
\text { rs7799039 }\end{array}$ & [65] \\
\hline \multirow[t]{2}{*}{ LEPR } & Leptin receptor & $\begin{array}{l}\text { rs1137100 } \\
\text { rs1137101 }\end{array}$ & {$[65,66]$} \\
\hline & & rs8179183 & [66] \\
\hline \multirow[t]{3}{*}{ GHRL } & Ghrelin & $\begin{array}{l}\text { rs696217 } \\
\text { rs4684677 }\end{array}$ & {$[65,67-69]$} \\
\hline & & rs34911341 & {$[68,69]$} \\
\hline & & rs2075356 & [69] \\
\hline \multirow[t]{4}{*}{ MC4R } & Melanocortin 4 receptor & & \\
\hline & & rs52820871 & {$[70,71]$} \\
\hline & & rs17782313 & {$[65,72]$} \\
\hline & & rs489693 & [65] \\
\hline \multirow[t]{2}{*}{ AGRP } & Agouti related protein & rs5030980 & {$[65,73]$} \\
\hline & & rs13338499 & [65] \\
\hline POMC & Proopiomelanocortin & rs 1042571 & [65] \\
\hline \multirow[t]{2}{*}{ ESR1 } & Estrogen receptor 1 & $\begin{array}{l}\text { rs726281 } \\
\text { rs22295193 } \\
\text { rs3798577 }\end{array}$ & [74] \\
\hline & & $\begin{array}{l}\text { rs2234693 } \\
\text { rs9340799 }\end{array}$ & [75] \\
\hline \multirow[t]{3}{*}{ ESR2 } & Estrogen receptor 2 & rs1256049 & {$[75,76]$} \\
\hline & & rs4986938 & [75] \\
\hline & & rs928554 & [77] \\
\hline \multirow[t]{2}{*}{ BDNF } & Brain-derived neurotrophic factor & rs6265 & {$[65,78,79]$} \\
\hline & & rs56164415 & {$[65,78]$} \\
\hline CNR1 & Cannabinoid receptor 1 & rs1049353 & {$[80,81]$} \\
\hline \multirow[t]{3}{*}{ OPRD1 } & Opioid receptor delta 1 & $\begin{array}{l}\text { rs536706 } \\
\text { rs760589 } \\
\text { rs204081 }\end{array}$ & [51] \\
\hline & & $\begin{array}{l}\text { rs569356 } \\
\text { rs521809 } \\
\text { rs4654327 }\end{array}$ & {$[52]$} \\
\hline & & $\begin{array}{l}\text { rs536706 } \\
\text { rs204081 }\end{array}$ & [51] \\
\hline
\end{tabular}


Table 2 Overview of candidate gene findings in eating disorders (Continued)

\begin{tabular}{|c|c|c|c|}
\hline Gene & Description & rs Number & References \\
\hline OPRM & Opioid receptor mu 1 & rs1799971 & [82] \\
\hline FTO & Fat mass and obesity associated & rs9939609 & {$[83,84]$} \\
\hline GHRL & Preproghrelin gene & $\begin{array}{l}\text { rs696217 } \\
\text { rs2075356 }\end{array}$ & [69] \\
\hline $\mathrm{TPH} 2$ & Tryptophan hydroxylase 2 & rs1473473 & [85] \\
\hline POMC & Pro-opiomelanocortin & rs24831852 & [86] \\
\hline NTRK3 & Neurotrophic tyrosine kinase receptor type 3 & rs7180942 & [87] \\
\hline FAAH & Fatty acid amide hydrolase & $\begin{array}{l}\text { rs932816 } \\
\text { rs324420 rs324419 } \\
\text { rs873978 rs2295632 }\end{array}$ & [80] \\
\hline NAAA & $\mathrm{N}$-acylethanolamine-hydrolyzing acid amidase & $\begin{array}{l}\text { rs2292534 rs4859567 } \\
\text { rs10518142 } \\
\text { rs6819442 }\end{array}$ & [80] \\
\hline MGLL & Monoglyceride lipase & rs893294 & [80] \\
\hline
\end{tabular}

\section{Molecular studies of eating disorder and obesity}

The objective of linkage studies is to find out the genomic regions which are more likely to contain genes implicated with a particular disease or trait. For linkage analysis, experiment is carried out on samples related to individuals (for example, dense pedigrees, affected sibling pairs, parent-offspring trios), and no priori hypotheses formed on the basis of priori data or biological function are required. Candidate-gene association studies, commonly abbreviated as CGAS, have been vastly effective for disorders where relationship between the selected gene and pathogenesis of disease is already well grounded [49]. In case of psychiatric disorders with completely elucidated underlying pathophysiology, candidate-gene approaches employ more speculation in regard to pertinence of the concerned gene, and therefore, have less prior possibility in identifying true association [49]. Candidate gene studies in the field of eating disorders need to take the following clinical observations into account: (A) The existence of $\mathrm{AN}$ and $\mathrm{BN}$ in female is remarkably greater (ratio 9:1). (B) The clinical manifestation stage of $\mathrm{BN}$ and $\mathrm{AN}$ appears more frequently in late adolescence and puberty respectively. (C) Around $30 \%$ of AN patients develop $\mathrm{BN}$ in future; the reverse direction is less common. (D) Increased comorbidity rate has been seen with major depression, generalized anxiety disorder, and obsessive-compulsive disorder (OCD) [50]. Candidate-gene studies which have obtained popularity for studying genetics of obesity and EDs mainly focus on single-nucleotide polymorphisms (SNPs) with priori hypotheses grounded on biological function (as expressed in animal, in vivo or in vitro studies). Table 2 will succinctly outline the highlights that are attained from candidate-gene studies on EDs. GWAS has been carried out on obesity and EDs in order to estimate genetic relationships between various disorder and physical characteristics. Genetic correlations were calculated significantly positive between AN and neuroticism, schizophrenia, high-density lipoprotein cholesterol, OCD, and educational attainment. On the contrary, genetic correlations were calculated significantly negative between AN and insulin sensitivity, body mass index (BMI), lipid phenotypes, and glucose metabolism [88, 89]. We have experienced 3 successful waves of innovations backed up by high-density large-scale GWAS for obesity-linked traits (Table 3). In most of the studies, SNPs have been tested for correlation with BMI or body mass index as a continuous trait $[97,111]$. BMI is regarded as a suitable proxy-measure of adult's adiposity which is quite convenient to obtain as well as available in numerous studies.

\section{Emerging hypotheses, clinical implications, and future directions Is dieting a risk for obesity?}

Regardless of the fact that many people tout dieting as a solution for the upheaval of obesity epidemic, some prospective studies concluded that dieting is ineffective in thwarting weight gain [112]. On top of that, recent study has also resurrected the idea stating as "dieting makes someone fat" [113]. Neumark-Sztainer et al. [114], Field et al. [115], and Stice et al. [116] are some of the most intriguing researches which suggest that adolescent girls who undergo dieting have increased likelihood in gaining weight and becoming obese compared to their nondieting companions. It is, however, somewhat ambiguous to decide if dieting is causal factor in this correlation, or could it be that, a largely futile strategy happened to come by people who tend to over-eat and gain weight. Evidence has been increasing in such a way that properly run and evaluated child obesity interventions reduce weight and pathology of eating disorder. This eventually 
Table 3 Common gene variants are associated with obesity phenotypes

\begin{tabular}{|c|c|c|c|}
\hline Gene & Description & rs Number & References \\
\hline FTO & Alpha-ketoglutarate-dependent dioxygenase & $\begin{array}{l}\text { rs9939609 } \\
\text { rs9930506 } \\
\text { rs1121980 } \\
\text { rs1421085 } \\
\text { rs8050136 rs1558902 } \\
\text { rs17817449 rs12149832 } \\
\text { rs9940128 } \\
\text { rs62033400 } \\
\text { rs9941349 } \\
\text { rs3751812 }\end{array}$ & {$[90,91]$} \\
\hline MC4R & Melanocortin 4 receptor & $\begin{array}{l}\text { rs } 17782313 \text { rs571312 } \\
\text { rs12970134 rs2331841 } \\
\text { rs6567160 rs } 8089364 \\
\text { rs7234864 rs723486 } \\
\text { rs7227255 rs2229616 } \\
\text { rs } 17782313 \text { rs17700144 } \\
\text { rs663129 rs571312 } \\
\text { rs } 476828\end{array}$ & {$[92,93]$} \\
\hline PCSK1 & Proprotein onvertase subtilisin/kexin type 1 & $\begin{array}{l}\text { rs6232 } \\
\text { rs6234 }\end{array}$ & [94] \\
\hline CTNNBL1 & Catenin beta like 1 & rs6013029 & [95] \\
\hline TMEM18 & Transmembrane protein 18 & $\begin{array}{l}\text { rs } 6548238 \\
\text { rs } 2867125 \\
\text { rs } 4854344 \text { rs } 7561317 \\
\text { rs } 11127485\end{array}$ & {$[91,96]$} \\
\hline GNPDA2 & Glucosamine-6-phosphate deaminase 2 & $\begin{array}{l}\text { rs10938397 } \\
\text { rs13130484 } \\
\text { rs348495 }\end{array}$ & {$[97,98]$} \\
\hline $\mathrm{SH} 2 \mathrm{~B} 1$ & Src homology 2B adapter protein 1 & rs7498665 & [97] \\
\hline KCTD15 & Potassium channel tetramerization domain containing 15 & $\begin{array}{l}\text { rs1 } 1084753 \\
\text { rs29941 }\end{array}$ & {$[91,97]$} \\
\hline $\mathrm{MTCH} 2$ & Mitochondrial carrier homolog 2 & $\begin{array}{l}\text { rs10838738 } \\
\text { rs3817334 }\end{array}$ & {$[97,98]$} \\
\hline NEGR1 & Neuronal growth regulator 1 & $\begin{array}{l}\text { rs2815752 rs3101336 } \\
\text { rs2568958 }\end{array}$ & {$[91,97]$} \\
\hline NPC1 & Niemann-pick disease, type C1 & rs1805081 & [99] \\
\hline MAF & MAF BZIP transcription factor & rs1424233 & [99] \\
\hline PTER & Phosphotriesterase related gene & rs10598503 & [99] \\
\hline PRL & Prolactin gene & rs4712652 & [99] \\
\hline SEC16B & Endoplasmic reticulum export factor & rs10913469 & [91] \\
\hline ETV5 & ETS variant 5 & rs7647305 rs9816226 & [91] \\
\hline AlF1 & Allograft inflammatory factor 1 & rs2844479 & [91] \\
\hline BDNF & Brain-derived neurotrophic factor & $\begin{array}{l}\text { rs6265 } \\
\text { rs } 4923461 \\
\text { rs10767664 rs2030323 } \\
\text { rs } 988712 \\
\text { rs925946 }\end{array}$ & [91] \\
\hline FAIM2 & Fas apoptotic inhibitory molecule 2 & rs7138803 rs7132908 & [91] \\
\hline SDCCAG8 & Serologically defined colon cancer antigen 8 & rs12145833 & [96] \\
\hline TNKS & Tankyrase & rs17150703 & [96] \\
\hline TFAP2B & Transcription factor AP-2 beta & $\begin{array}{l}\text { rs } 987237 \\
\text { rs } 987237 \text { rs734597 } \\
\text { rs } 2272903\end{array}$ & [98] \\
\hline MSRA & Methionine sulfoxide reductase $\mathrm{A}$ & rs7826222 & [100] \\
\hline LYPLAL1 & Lysophospholipase-like 1 & rs4846567 & [100] \\
\hline
\end{tabular}


Table 3 Common gene variants are associated with obesity phenotypes (Continued)

\begin{tabular}{|c|c|c|c|}
\hline Gene & Description & rs Number & References \\
\hline NRXN3 & Neurexin 3 & $\begin{array}{l}\text { rs10146997 } \\
\text { rs10150332 }\end{array}$ & {$[98,101]$} \\
\hline HECTD4 & HECT domain E3 ubiquitin protein ligase 4 & rs2074356 & [102] \\
\hline GPRC5BB & G protein-coupled receptor, class $C$, group 5 , member $\mathrm{Bb}$ & rs12444979 & [103] \\
\hline POMC & Pro-opiomelanocortin & $\begin{array}{l}\text { rs713586 } \\
\text { rs6545814 } \\
\text { rs1561288 rs6752378 } \\
\text { rs10182181 }\end{array}$ & {$[98,103]$} \\
\hline MAP2K5 & Mitogen-activated protein kinase 5 & $\begin{array}{l}\text { rs } 2241423 \\
\text { rs } 2241423 \text { rs4776970 } \\
\text { rs } 997295\end{array}$ & {$[98,103]$} \\
\hline GIPR & Gastric inhibitory polypeptide receptor & $\begin{array}{l}\text { rs2287019 } \\
\text { s11671664 }\end{array}$ & [103] \\
\hline FANCL & Fanconi anemia complementation group $L$ & $\begin{array}{l}\text { rs887912 } \\
\text { rs12617233 }\end{array}$ & [103] \\
\hline TNNI3K & TNNI3 interacting kinase & $\begin{array}{l}\text { rs } 1514175 \\
\text { rs12142020 } \\
\text { rs1040070 rs1514174 }\end{array}$ & {$[98,103]$} \\
\hline TMEM160 & Transmembrane protein 160 & rs3810291 & [103] \\
\hline CADM2 & Cell adhesion molecule 2 & rs13078807 & [103] \\
\hline LRP1B & Low-density lipoprotein receptor & rs2890652 & [103] \\
\hline PRKD1 & Protein kinase D1 & $\begin{array}{l}\text { rs11847697 } \\
\text { rs12885454 }\end{array}$ & [103] \\
\hline MTIF3 & Mitochondrial translational initiation factor 3 & rs4771122 & [103] \\
\hline ZNF608 & Zinc finger protein 608 & rs48361333 & [103] \\
\hline PTBP2 & Polypyrimidine tract binding protein 2 & rs1555543 & [103] \\
\hline TUB & Tubby protein homolog & rs4929949 & [103] \\
\hline HMGA1 & High mobility group AT-hook 1 & rs206936 & [103] \\
\hline MC4R & Melanocortin 4 receptor & rs7227255 & [103] \\
\hline RSPO3 & R-spondin 3 & rs9491696 & [104] \\
\hline VEGFA & Vascular endothelial growth factor A & rs6905288 & [104] \\
\hline TBX15 & T-box transcription factor 15 & rs984222 & [104] \\
\hline NFE2L3 & Nuclear factor erythroid 2-like factor 3 & rs1055144 & [104] \\
\hline GRB14 & Growth factor receptor bound protein 14 & rs10195252 & [104] \\
\hline DNM3 & Dynamin-3 & rs1011731 & [104] \\
\hline ITPR2/SSPN & Inositol 1,4,5-trisphosphate receptor type 2 & rs718314 & [104] \\
\hline LY86 & Lymphocyte antigen 86 & rs1294421 & [104] \\
\hline $\mathrm{HOXC13}$ & Homeobox protein hox-C13 & rs1443512 & [104] \\
\hline ADAMTS9 & A disintegrin and metalloproteinase with thrombospondin motifs 9 & rs6795735 & [104] \\
\hline ZNRF3 & Zinc and ring finger 3 & rs4823006 & [104] \\
\hline $\mathrm{NISCH}$ & Nischarin gene & rs6784615 & [104] \\
\hline CPEB4 & Cytoplasmic polyadenylation element binding protein 4 & rs6861681 & [104] \\
\hline RASAL2 & RAS protein activator like 2 & $\begin{array}{l}\text { rs10913469 } \\
\text { rs543874 } \\
\text { rs574367 rs516636 } \\
\text { rs591120 }\end{array}$ & {$[91,103]$} \\
\hline LYPLAL1 & Lysophospholipase-like protein 1 & rs2605100 & [104] \\
\hline SLC39A8 & Solute carrier family 39 member 8 & rs13107325 & [103] \\
\hline NCR3 & Natural cytotoxicity triggering receptor 3 & rs2844479 rs2260000 & [31] \\
\hline
\end{tabular}


Table 3 Common gene variants are associated with obesity phenotypes (Continued)

\begin{tabular}{|c|c|c|c|}
\hline Gene & Description & rs Number & References \\
\hline & & rs1077393 & \\
\hline NUDT3 & Nudix hydrolase 3 & rs206936 & [103] \\
\hline RSPOS & roof plate-specific spondin & rs9491696 & [104] \\
\hline RPL27A & Ribosomal protein L27a & rs4929949 & [103] \\
\hline ITPR2 & Nositol 1,4,5-trisphosphate receptor, type 2 & rs718314 & [104] \\
\hline FAIM2 & Fas apoptotic inhibitory molecule 2 & rs7138803 & [91] \\
\hline MTIF3 & Mitochondrial translational initiation factor 3 & rs4771122 & [103] \\
\hline NRXN3 & Neurexin 3 & rs10146997 & [101] \\
\hline $\mathrm{SH} 2 \mathrm{~B} 1$ & SH2B adaptor protein 1 & $\begin{array}{l}\text { rs7498665 rs8049439 } \\
\text { rs4788102 }\end{array}$ & [91] \\
\hline MC4R & Melanocortin 4 receptor & $\begin{array}{l}\text { rs } 17782313 \text { rs12970134 } \\
\text { rs17700144 }\end{array}$ & {$[91,103]$} \\
\hline GIPR & Gastric inhibitory polypeptide receptor & rs2287019 & [103] \\
\hline TMEM18 & Transmembrane Protein 18 & $\begin{array}{l}\text { rs2867125 } \\
\text { rs6548238 rs7561317 } \\
\text { rs12463617 } \\
\text { rs } 4854344\end{array}$ & {$[91,103]$} \\
\hline QPCTL & Glutaminyl-peptide cyclotransferase like & rs2287019 & {$[91,103]$} \\
\hline ADCY9 & Adenylate cyclase 9 & rs2531995 & [98] \\
\hline AGBL4 & ATP/GTP binding protein like 4 & rs657452 & [105] \\
\hline ASB4 & Ankyrin repeat and SOCS box containing 4 & rs6465468 & [105] \\
\hline CCDC171 & Coiled-coil domain containing 171 & rs4740619 & [105] \\
\hline CADM1 & Cell adhesion molecule 1 & rs12286929 & [105] \\
\hline CADM2 & Cell adhesion molecule 2 & rs13078807 & [103] \\
\hline CALCR & Calcitonin receptor & rs9641123 & [105] \\
\hline CBLN1 & Cerebellin 1 precursor & rs2080454 & [105] \\
\hline CDKAL1 & CDK5 regulatory subunit associated protein 1 like 1 & rs2206734, rs9356744 & [106] \\
\hline CLIP1 & CAP-Gly domain containing linker protein 1 & rs11057405 & {$[105]$} \\
\hline CREB1 & CAMP responsive element binding protein 1 & rs17203016 & [105] \\
\hline EHBP1 & EH domain binding protein 1 & rs11688816 & [105] \\
\hline ELAVL4 & ELAV like RNA binding protein 4 & rs11583200 & [105] \\
\hline EPB41L4B & Erythrocyte membrane protein band 4.1 like $4 \mathrm{~B}$ & rs6477694 & [105] \\
\hline ERBB4 & Erb-B2 receptor tyrosine kinase 4 & rs7599312 & {$[105]$} \\
\hline ETS2 & ETS proto-oncogene 2 & rs2836754 & [105] \\
\hline FIGN & Fidgetin, microtubule severing factor & rs1460676 & {$[105]$} \\
\hline FHIT & Fragile histidine triad protein & rs2365389 & [105] \\
\hline FOXO3 & Forkhead box $\mathrm{O} 3$ & rs9400239 & [105] \\
\hline GBE1 & 1,4-Alpha-glucan branching enzyme 1 & rs3849570 & [105] \\
\hline GDF15 & Growth/differentiation factor 15 & rs17724992 & [105] \\
\hline GNAT2 & G protein subunit alpha transducin 2 & rs17024258 & [98] \\
\hline GP2 & Glycoprotein 2 & rs12597579 & {$[106]$} \\
\hline GRID1 & Glutamate ionotropic receptor delta type subunit 1 & rs7899106 & [105] \\
\hline GRP & Gastrin releasing peptide & rs7243357 & [105] \\
\hline GRP120 & G-protein coupled receptor 120 & rs116454156 & [107] \\
\hline HHIP & Hedgehog interacting protein & rs11727676 & {$[105]$} \\
\hline HIF1AN & Hypoxia inducible factor 1 subunit alpha inhibitor & rs17094222 & [105] \\
\hline
\end{tabular}


Table 3 Common gene variants are associated with obesity phenotypes (Continued)

\begin{tabular}{|c|c|c|c|}
\hline Gene & Description & rs Number & References \\
\hline HIP1 & Huntingtin Interacting Protein 1 & rs1167827 & [105] \\
\hline HMGA1 & High mobility group AT-hook 1 & rs206936 & [103] \\
\hline HNF4G & Hepatocyte nuclear factor 4 Gamma & rs4735692 & [98] \\
\hline HOXB5 & Homeobox B5 & rs9299 & [106] \\
\hline HS6ST3 & Heparan sulfate sulfotransferases & rs7989336 & [98] \\
\hline HSD17B12 & Hydroxysteroid 17-beta dehydrogenase 12 & rs2176598 & [105] \\
\hline IFNGR1 & Interferon gamma receptor 1 & rs13201877 & [105] \\
\hline KAT8 & Lysine acetyltransferase 8 & rs9925964 & [105] \\
\hline KCNK3 & Potassium two pore domain channel subfamily $\mathrm{K}$ member 3 & rs11126666 & [105] \\
\hline KCNMA1 & Potassium calcium-activated channel subfamily M alpha 1 & rs2116830 & [108] \\
\hline KLF9 & Kruppel like factor 9 & rs11142387 & [109] \\
\hline LEPR & Leptin receptor & rs11208659 & [110] \\
\hline LMX1B & LIM homeobox transcription factor 1-beta & rs10733682 & [105] \\
\hline BBS4 & Bardet-biedl syndrome 4 & rs7164727 & [105] \\
\hline RIT2 & GTP-binding protein Rit2 & rs7239883 & [105] \\
\hline MAPK3 & Mitogen-activated protein kinase 3 & rs4787491 & [105] \\
\hline MIR548A2 & MicroRNA 548a-2 & $\begin{array}{l}\text { rs1441264 } \\
\text { rs9540493 }\end{array}$ & [105] \\
\hline MRPS33P4 & mitochondrial ribosomal protein S33 pseudogene 4 & rs13041126 & [98] \\
\hline NAV1 & Neuron navigator 1 & rs2820292 & [105] \\
\hline NLRC3 & NOD-like receptor family CARD domain containing 3 & rs758747 & [105] \\
\hline NT5C2 & 5'-Nucleotidase, cytosolic II & rs11191560 & [105] \\
\hline NTRK2 & Neurotrophic receptor tyrosine kinase 2 & rs1211166 & [105] \\
\hline NUP54 & Nucleoporin 54 & rs17001654 & [105] \\
\hline OLFM4 & Olfactomedin 4 & $\begin{array}{l}\text { rs9568856 } \\
\text { rs9568867 }\end{array}$ & [98] \\
\hline PACS1 & Phosphofurin acidic cluster sorting protein 1 & rs564343 & [110] \\
\hline PARK2 & parkin RBR E3 ubiquitin protein ligase & rs13191362 & [99] \\
\hline PLCD4 & Phospholipase $C$ delta 4 & rs492400 & [99] \\
\hline PMS2L11 & postmeiotic segregation increased 2-like 11 & rs2245368 & [99] \\
\hline $\mathrm{PRKCH}$ & Protein kinase C Eta & rs1957894 & [110] \\
\hline RABEP1 & Rabaptin, RAB GTPase binding effector protein 1 & rs1000940 & [105] \\
\hline RALYL & RALY RNA binding protein like & rs2033732 & [105] \\
\hline RARB & Retinoic acid receptor beta & rs6804842 & [105] \\
\hline RASA2 & RAS P21 protein activator 2 & rs16851483 & [105] \\
\hline RMST & Rhabdomyosarcoma 2 associated transcript & rs11109072 & [110] \\
\hline RPTOR & Regulatory associated protein Of MTOR complex 1 & rs7503807 & [98] \\
\hline SBK1 & SH3 domain binding kinase 1 & rs2650492 & [105] \\
\hline DMXL2 & Dmx-like 2 & rs3736485 & [105] \\
\hline SMG6 & SMG6 nonsense mediated MRNA decay factor & rs9914578 & [105] \\
\hline STXBP6 & Syntaxin-binding protein 6 & rs10132280 & [105] \\
\hline TAL1 & T-cell acute lymphocytic leukemia protein 1 & rs977747 & [105] \\
\hline TCF7L2 & Transcription factor 7-like 2 & rs7903146 & [105] \\
\hline TDRG1 & Testis development related 1 & rs2033529 & [105] \\
\hline TLR4 & Toll-like receptor 4 & rs1928295 & [105] \\
\hline
\end{tabular}


Table $\mathbf{3}$ Common gene variants are associated with obesity phenotypes (Continued)

\begin{tabular}{llll}
\hline Gene & Description & rs Number & References \\
\hline TOMM40 & Translocase of outer mitochondrial membrane 40 homolog & rs2075650 & [106] \\
UBE2E3 & Ubiquitin conjugating enzyme E2 E3 & rs1528435 & [105] \\
ZBTB10 & Zinc finger and BTB domain containing 10 & rs16907751 & [105] \\
ZZZ3 & Zinc finger ZZ-type containing 3 & rs17381664 & [98] \\
\hline
\end{tabular}

makes work on improving and shaping up the dieting conceptualization an even more priority.

\section{Management of obesity-implications for eating disorders}

Many people, especially the health professionals who are engaged in treating obese and overweight children have frequently voiced their concern over the issue that active involvement in weight loss can sometimes lead to the development of an eating disorder in children (Fig. 2). This hurdle has been hard to leap over, since there have not been enough number of treatments regarding chronic child obesity in the literature. These particular children have been supervised for a period of 10 years or above, and over the time, eating disorders have been occasionally occurring in children who underwent these programs, with the rates close to those estimated in the community. In 2005, the review of a literature by Butryn and Brownwell found 5 researches related to this [117]. They summarized by mentioning that children and teenagers participating in weight-loss programs that were administered professionally did not have increased eating disorders symptoms. In fact, they were observed to develop significantly improved psychological well-being among them [118]. Thus, weight control management in no way carries hazard for eating disorders if done expertly and properly, but in contrast, it could be considered a risk without the presence of this control and organization. Secondly, as some particular groups of overweight teenagers and children are more prone to eating disorders than others, more attention are in need to explore these underlying risks and the method how those risks can be screened. Already, the propensity for launching negative health campaign on obesity has been raised with a view to mobilizing public to alter their behavior. Hence, any attempt that is likely to further stigmatize fat people and obesity may ferment adolescent and child weight concerns and thereby risking the increase of eating disorders [119].

\section{Joint working in future}

Both eating disorders and obesity would reap benefits from an education that teaches the malefactor aspect of both disorders. Women belonged to the high risk group for eating disorders might not have judged their physical outlook so desperately if the perceived value of being slim had not been so great. A common perspective that obese people should look at their weight negatively to stimulate them with a view to reducing bulkiness, is misplaced. It is a matter of fact that most people find it exceedingly hard to bring a huge and long-lasting transformation of their weight, and a high level of discontentment is not supposed to guarantee success for sure. Rather, a personal perception of futility and failure may be added in attempting to lose weight. Modest loss of weight $(10 \%)$, as recommended by the heath professional, can paradoxically be achieved by depleting the negative delineation of obesity. Usually, personally taken goals for weight loss end up largely being in smoke, making people finally to give up. In the survey, there has been no mention of any statement concerning the weight loss behavior that the overweight people are giving more concentrated effort with the obese people

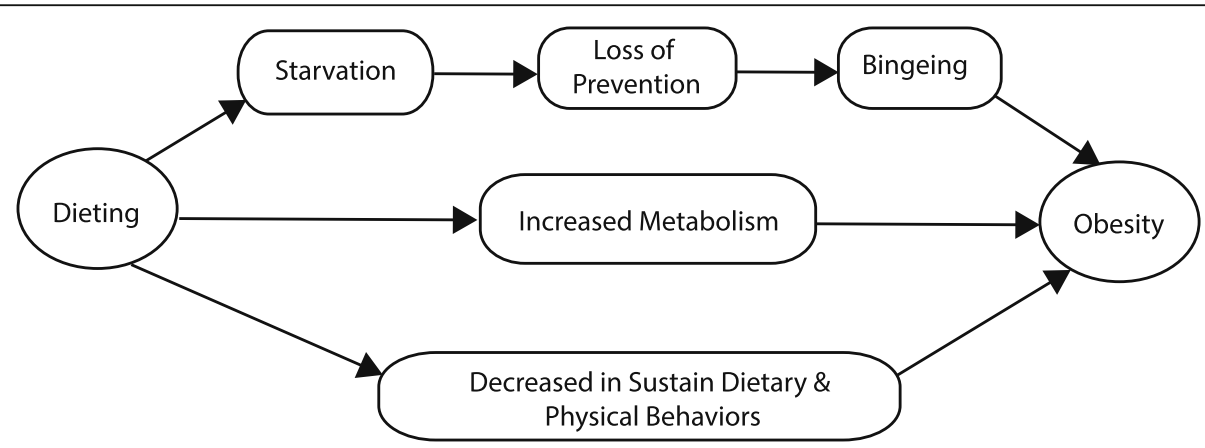

Fig. 2 Hypothesized associations and interrelationship between dieting and obesity 
being less involved when it comes to trying. If it had been possible to make public understand about the intricacy of weight loss and weight regulation, enormous benefits would have obtained. Despite the truth that psycho-educational strategy is central for the treatments of numerous eating disorders, it has been disparaged in managing obesity. Without focusing on energy balance, an oversimplified message expressed in a phrase "Exercise more and eat less" is now widespread. We would not experience the present obesity levels if the solution was that facile. Therefore, the collaboration of the government, media, and health care professionals is an urgency to disseminate helpful, realistic, and consistent information [120]. Promotion of self-esteem in teenager and children should be one area to focus. This may also impart benefits to other health issues including conduct disorder and self-harm. Alternatively, surroundings that encourage and make young people eating healthily can be of mutual advantages to eating disorders and obesity. Therefore additional proof of long-term advantages of suchlike interventions is required.

\section{Conclusion}

Obesity and eating disorder perturb all ages, races, gender, sexual orientation, and ethnicities. People who suffer from health disorder or certain mental behavior problem undergo further complex medical condition later in their life. There are some particular genotypes which can make people more vulnerable to acquire those disorders. This review document explicated the complex genotypic correlation of eating disorders with obesity and overweight. It also delineated clinical implications, promising hypotheses, and future directions. It is high time we should talk and clarify it. Since this is a public health concern, making people well understand will definitely direct them through the exact way of tackling this epidemic. If early intervention, apposite treatment, and proper management are ensured, it is possible to gain complete recovery from this mental disorder.

\section{Abbreviations}

EDs: Eating disorders; BED: Binge eating disorder; BMI: Body mass index AN: Anorexia nervosa; BN: Bulimia nervosa; GWASs: Genome-wide association studies

\section{Acknowledgements}

The authors would like to express their heartfelt obligation, indebtedness, gramercy, and profound appreciation are made to Department of Pharmacy, Comilla University, Comilla-3506, Bangladesh, Department of Pharmacy, Noakhali Science and Technology University, Sonapur-3814, Noakhali, Bangladesh and Department of Pharmacy, University of Chittagong, Chittagong-4331, Bangladesh for their general facilities continuous support, untiring inspiration, scholastic supervision, constructive criticism, affectionate feeling, and optimistic counseling for the conduction of this study.

\section{Authors' contributions}

SKA and $A B$ equally participated in the conduction of the review. AFM, MK, and MSR did the initial literature search. UKR and AB participated in preparing the manuscript. SMNU designed the concept, analyzed the data.
MAA redesigned the concept. SMNU and MAA revised the manuscript. All authors read and approved the final manuscript.

Funding

There have no funding for this review work.

Availability of data and materials

Not applicable.

\section{Declarations}

Ethics approval and consent to participate

Not applicable.

\section{Consent for publication}

Not applicable.

\section{Competing interests}

The authors declare that they have no competing interests.

\section{Author details}

${ }^{1}$ Department of Pharmacy, Comilla University, Comilla 3506, Bangladesh. ${ }^{2}$ Department of Pharmacy, Noakhali Science and Technology University, Sonapur, Noakhali 3814, Bangladesh. ${ }^{3}$ Department of Genetic Engineering and Biotechnology, University of Chittagong, Chittagong 4331, Bangladesh. ${ }^{4}$ Department of Pharmacy, University of Chittagong, Chittagong 4331, Bangladesh.

Received: 22 April 2020 Accepted: 19 April 2021

Published online: 26 May 2021

\section{References}

1. Qian J, Hu Q, Wan Y, Li T, Wu M, Ren Z, Yu D (2013) Prevalence of eating disorders in the general population: a systematic review. Shanghai Arch Psychiatry 25(4):212-223. https://doi.org/10.3969/j.issn.1002-0829.2013.04.003

2. Lyons L, Ekern J (2019) Eating disorders on the rise all around the world: an overview. Eating Disorder Hope. https://www.eatingdisorderhope.com/blog/ eating-disorders-world-overview. Accessed 12 Nov 2019.

3. Crow SJ, Swanson S (2014) Facts about eating disorders: what the research shows. https://www.emilyprogram.com/wp-content/uploads/edc-factsheet-2019.pdf. Accessed 12 Nov 2019.

4. Smink FR, van Hoeken D, Hoek HW (2012) Epidemiology of eating disorders: incidence, prevalence and mortality rates. Curr Psychiatry Rep 14(4):406-414. https://doi.org/10.1007/s11920-012-0282-y

5. Makino M, Tsuboi K, Dennerstein L (2004) Prevalence of eating disorders: a comparison of Western and non-Western countries. MedGenMed 6(3):49

6. Erskine HE, Whiteford HA, Pike KM (2016) The global burden of eating disorders. Curr Opin Psychiatry 29(6):346-353. https://doi.org/10.1097/YCO. 0000000000000276

7. Al-Adawi S, Dorvlo AS, Burke DT et al (2002) A survey of anorexia nervosa using the Arabic version of the EAT-26 and "gold standard" interviews among Omani adolescents. Eat Weight Disord 7(4):304-311. https://doi. org/10.1007/bf03324977

8. Thomas JJ, Lee S, Becker AE (2016) Updates in the epidemiology of eating disorders in Asia and the Pacific. Curr Opinion Psychiatry 29(6):354-362. https://doi.org/10.1097/YCO.0000000000000288

9. Becker AE (2004) Television, disordered eating, and young women in Fiji: negotiating body image and identity during rapid social change. Cult Med Psychiatry 28(4):533-559. https://doi.org/10.1007/s11013-004-1067-5

10. Pike KM, Dunne PE (2015) The rise of eating disorders in Asia: a review. J Eat Disord 3(1):33. https://doi.org/10.1186/s40337-015-0070-2

11. Lee $S$ (2004) Engaging culture: an overdue task for eating disorders research. Cult Med Psychiatry 28(4):617-621. https://doi.org/10.1007/s11013004-1072-8

12. Moussa HN, Alrais MA, Leon MG, Abbas EL, Sibai BM (2016) Obesity epidemic: impact from preconception to postpartum. Future Sci 2(3): FSO137. https://doi.org/10.4155/fsoa-2016-0035

13. Rusek W, Baran J, Leszczak J, Adamczyk M, Weres A, Baran R, Inglot G, Pop T (2018) The Influence of Body Mass Composition on the Postural Characterization of School-Age Children and Adolescents. Biomed Res Int 2018:9459014-9459017. https://doi.org/10.1155/2018/9459014 
14. Deren K, Nyankovskyy S, Nyankovska O et al (2018) The prevalence of underweight, overweight and obesity in children and adolescents from Ukraine. Sci Rep 8(1):3625. https://doi.org/10.1038/s41598-018-21 $773-4$

15. Jafar TH, Qadri Z, Islam M, Hatcher J, Bhutta ZA, Chaturvedi N (2007) Rise in childhood obesity with persistently high rates of undernutrition among urban school-aged Indo-Asian children. Arch Dis Child 93(5):373-378. https://doi.org/10.1136/adc.2007.125641

16. Zamsad M, Banik S, Ghosh L (2019) Prevalence of overweight, obesity and abdominal obesity in Bangladeshi university students: A cross-sectional study. Diabetes Metab Syndrome 13(1):480-483. https://doi.org/10.1016/j. dsx.2018.11.015

17. Magallares A, Pais-Ribeiro $J$ (2013) Mental Health and Obesity: A MetaAnalysis. Appl Res Qual Life 9(2):295-308. https://doi.org/10.1007/s11482013-9226-x

18. Tronieri JS, Wurst CM, Pearl RL, Allison KC (2017) Sex Differences in Obesity and Mental Health. Curr Psychiatry Rep 19(6):29. https://doi.org/10.1007/s11 920-017-0784-8

19. Biswas T, Islam A, Islam MS, Pervin S, Rawal LB (2017) Overweight and obesity among children and adolescents in Bangladesh: a systematic review and meta-analysis. Public Health 142:94-101. https://doi.org/10.1016/j. puhe.2016.10.010

20. Pengpid S, Peltzer K, Ahsan GU (2015) Risk of eating disorders among university students in Bangladesh. Int J Adolescent Med Health 27(1):93100. https://doi.org/10.1515/ijamh-2014-0013

21. Treasure J, Zipfel S, Micali N, Wade T, Stice E, Claudino A, Schmidt U, Frank GK, Bulik CM, Wentz E (2015) Anorexia nervosa. Nat Rev Dis Prim 1(1):15074. https://doi.org/10.1038/nrdp.2015.74

22. Hoek HW (2006) Incidence, prevalence and mortality of anorexia nervosa and other eating disorders. Curr Opin Psychiatry 19(4):389-394. https://doi. org/10.1097/01.yco.0000228759.95237.78

23. Harrington BC, Jimerson M, Haxton C, Jimerson DC (2015) Initial evaluation, diagnosis, and treatment of anorexia nervosa and bulimia nervosa. Am Fam Physician 91(1):46-52

24. Attia E, Haiman C, Walsh BT, Flater SR (1998) Does fluoxetine augment the inpatient treatment of anorexia nervosa? Am J Psychiatry 155(4):548-551. https://doi.org/10.1176/ajp.155.4.548

25. Halmi KA, Eckert E, LaDu TJ, Cohen J (1986) Anorexia nervosa. Treatment efficacy of cyproheptadine and amitriptyline. Arch Gen Psychiatry 43(2):177181. https://doi.org/10.1001/archpsyc.1986.01800020087011

26. Biederman J, Herzog DB, Rivinus TM, Harper GP, Ferber RA, Rosenbaum JF, Harmatz JS, Tondorf R, Orsulak PJ, Schildkraut JJ (1985) Amitriptyline in the treatment of anorexia nervosa: a double-blind, placebo-controlled study. J Clin Psychopharmacol 5(1):10-16

27. Malina A, Gaskill J, McConaha C, Frank GK, LaVia M, Scholar L, Kaye WH (2003) Olanzapine treatment of anorexia nervosa: a retrospective study. Int J Eat Disord 33(2):234-237. https://doi.org/10.1002/eat.10122

28. Miller KK, Grieco KA, Klibanski A (2005) Testosterone administration in women with anorexia nervosa. J Clin Endocrinol Metab 90(3):1428-1433. https://doi.org/10.1210/jc.2004-1181

29. McElroy SL, Guerdjikova Al, Martens B et al (2009) Role of antiepileptic drugs in the management of eating disorders. CNS drugs 23(2):139-156. https:// doi.org/10.2165/00023210-200923020-00004

30. Birmingham CL, Goldner EM, Bakan R (1994) Controlled trial of zinc supplementation in anorexia nervosa. Int J Eat Disord 15(3):251-255

31. Pike KM, Walsh BT, Vitousek K, Wilson GT, Bauer J (2003) Cognitive behavior therapy in the posthospitalization treatment of anorexia nervosa. Am J Psychiatry 160(11):2046-2049. https://doi.org/10.1176/appi.ajp.160.11.2046

32. Dare C, Eisler I, Russell G, Treasure J, Dodge L (2001) Psychological therapies for adults with anorexia nervosa: randomised controlled trial of out-patient treatments. The Br J Psychiatry 178(3):216-221. https://doi.org/10.1192/bjp.1 78.3.216

33. Geist R, Heinmaa M, Stephens D, Davis R, Katzman DK (2000) Comparison of family therapy and family group psychoeducation in adolescents with anorexia nervosa. Can J Psychiatry 45(2):173-178. https://doi.org/10.1177/ 070674370004500208

34. Johnson CL, Taylor C (1996) Working with difficult-to-treat eating disorders using an integration of twelve-step and traditional psychotherapies. Psychiatric Clin North Am 19(4):829-841. https://doi.org/10.1016/s0193-953 x(05)70384-1

35. Hay PJ, Claudino AM (2010) Bulimia nervosa. BMJ Clin Evid 2010:1009
36. Levinson CA, Zerwas S, Calebs B, Forbush K, Kordy H, Watson H, Hofmeier S, Levine M, Crosby RD, Peat C, Runfola CD, Zimmer B, Moesner M, Marcus MD, Bulik CM (2017) The core symptoms of bulimia nervosa, anxiety, and depression: A network analysis. J Abnorm Psychol 126(3):340-354. https:// doi.org/10.1037/abn0000254

37. Chakraborty K, Basu D (2010) Management of anorexia and bulimia nervosa: an evidence-based review. Indian J Psychiatry 52(2):174-186. https://doi. org/10.4103/0019-5545.64596

38. Rushing JM, Jones LE, Carney CP (2003) Bulimia Nervosa: A Primary Care Review. Primary Care Companion J Clin Psychiatry 5(5):217-224. https://doi. org/10.4088/pcc.v05n0505

39. Becker AE (2003) Outpatient management of eating disorders in adults. Curr Womens Health Rep 3(3):221-229

40. Amianto F, Ottone L, Abbate Daga G, Fassino S (2015) Binge-eating disorder diagnosis and treatment: a recap in front of DSM-5. BMC Psychiatry 15(1):70. https://doi.org/10.1186/s12888-015-0445-6

41. Brownley KA, Berkman ND, Peat CM, Lohr KN, Bulik CM (2017) Binge-Eating Disorder in Adults. Ann Intern Med 166(3):231-232. https://doi.org/10.7326/ L16-0621

42. Wilfley DE, Fitzsimmons-Craft EE, Eichen DM (2017) Binge-eating disorder in adults. Ann Intern Med 166(3):230-231. https://doi.org/10.7326/L16-0622

43. Papp I, Szumska I, Tury F (2015) The treatment of binge eating disorder - a review. Psychiatria Hungarica 30(3):268-277

44. McElroy SL, Hudson Jl, Malhotra S et al (2003) Citalopram in the treatment of binge-eating disorder: a placebo-controlled trial. J Clin Psychiatry 64(7): 807-813. https://doi.org/10.4088/jcp.v64n0711

45. Pardo MDMA, Martin MF, Hernandez MAC, Marquez FM (2017) Adherence of psychopharmacological prescriptions to clinical practice guidelines in patients with eating behavior disorders. Eur J Clin Pharmacol 73(10):13051313. https://doi.org/10.1007/s00228-017-2287-2

46. Dingemans AE, Bruna MJ, Van Furth EF (2002) Binge eating disorder: a review. Int J Obes Relat Metab Disord 26(3):299-307. https://doi.org/10.103 8/sj.ijo.0801949

47. Devlin MJ, Goldfein JA, Dobrow I (2003) What is this thing called BED? Curr Status Binge Eat Disord Nosol 34 Suppl:S2-S18. https:/doi.org/10.1002/eat.10201

48. Yanovski SZ (2003) Binge eating disorder and obesity in 2003: could treating an eating disorder have a positive effect on the obesity epidemic? Int J Eat Disord 34(Suppl):S117-S120. https://doi.org/10.1002/eat.10211

49. McCarroll SA, Feng G, Hyman SE (2014) Genome-scale neurogenetics: methodology and meaning. Nat Neurosci 17(6):756-763. https://doi.org/10.1 038/nn.3716

50. Kaye WH, Klump KL, Frank GK, Strober M (2000) Anorexia and bulimia nervosa. Annu Rev Med 51(1):299-313. https://doi.org/10.1146/annurev.med. 51.1.299

51. Bergen AW, Bree MBVD, Yeager M et al (2003) Candidate genes for anorexia nervosa in the 1p33-36 linkage region: serotonin 1D and delta opioid receptor loci exhibit significant association to anorexia nervosa. Mol Psychiatry 8(4):397-406. https://doi.org/10.1038/sj.mp.4001318

52. Brown KM, Bujac SR, Mann ET et al (2007) Further evidence of association of OPRD1 \& HTR1D polymorphisms with susceptibility to anorexia nervosa. Biol Psychiatry 61(3):367-373. https://doi.org/10.1016/j.biopsych.2006.04.007

53. Ricca V, Nacmias B, Boldrini M, Cellini E, di Bernardo M, Ravaldi C, Tedde A, Bagnoli S, Placidi GF, Rotella CM, Sorbi S (2004) Psychopathological traits and 5-HT2A receptor promoter polymorphism (-1438 G/A) in patients suffering from Anorexia Nervosa and Bulimia Nervosa. Neurosci Lett 365(2): 92-96. https://doi.org/10.1016/j.neulet.2004.04.057

54. Martaskova D, Slachtova L, Kemlink D et al (2009) Polymorphisms in serotonin-related genes in anorexia nervosa. The first study in Czech population and metaanalyses with previously performed studies. Folia Biol 55(5):192-197

55. Enoch MA, Kaye WH, Rotondo A, Greenberg BD, Murphy DL, Goldman D (1998) 5-HT2A promoter polymorphism -1438G/A, anorexia nervosa, and obsessive-compulsive disorder. Lancet 351(9118):1785-1786. https://doi. org/10.1016/S0140-6736(05)78746-8

56. Hinney A, Ziegler A, Nothen MM et al (1997) 5-HT2A receptor gene polymorphisms, anorexia nervosa, and obesity. Lancet 350(9087):1324-1325. https://doi.org/10.1016/S0140-6736(05)62485-3

57. Hu X, Giotakis O, Li T, Karwautz A, Treasure J, Collier DA (2003) Association of the $5-\mathrm{HT} 2 \mathrm{C}$ gene with susceptibility and minimum body mass index in anorexia nervosa. Neuroreport 14(6):781-783. https://doi.org/10.1097/00001 756-200305060-00001 
58. Bergen AW, Yeager M, Welch RA, Haque K, Ganjei JK, van den Bree MBM, Mazzanti C, Nardi I, Fichter MM, Halmi KA, Kaplan AS, Strober M, Treasure J, Woodside DB, Bulik CM, Bacanu SA, Devlin B, Berrettini WH, Goldman D, Kaye WH (2005) Association of multiple DRD2 polymorphisms with anorexia nervosa. Neuropsychopharmacology 30(9):1703-1710. https://doi.org/10.103 8/sj.npp.1300719

59. Davis C, Levitan RD, Kaplan AS, Carter J, Reid C, Curtis C, Patte K, Hwang R, Kennedy JL (2008) Reward sensitivity and the D2 dopamine receptor gene: A case-control study of binge eating disorder. Prog Neuro-Psychopharmacol Biol Psychiatry 32(3):620-628. https://doi.org/10.1016/j.pnpbp.2007.09.024

60. Davis C, Levitan RD, Yilmaz Z, Kaplan AS, Carter JC, Kennedy JL (2012) Binge eating disorder and the dopamine D2 receptor: genotypes and subphenotypes. Prog Neuro-Psychopharmacol Biol Psychiatry 38(2):328-335. https://doi.org/10.1016/j.pnpbp.2012.05.002

61. Bruins-Slot L, Gorwood P, Bouvard M, Blot P, Adès J, Feingold J, Schwartz JC, Mouren-Siméoni MC (1998) Lack of association between anorexia nervosa and D3 dopamine receptor gene. Biol Psychiatry 43(1):76-78. https://doi. org/10.1016/S0006-3223(97)00199-6

62. Bachner-Melman R, Lerer E, Zohar AH, Kremer I, Elizur Y, Nemanov L, Golan M, Blank S, Gritsenko I, Ebstein RP (2007) Anorexia nervosa, perfectionism, and dopamine D4 receptor (DRD4). Am J Med Genetics Part B Neuropsychiatric Genetics 144B(6):748-756. https://doi.org/10.1002/ajmg.b.30505

63. Mikolajczyk E, Smiarowska M, Grzywacz A, Samochowiec J (2006) Association of eating disorders with catechol-o-methyltransferase gene functional polymorphism. Neuropsychobiology 54(1):82-86. https://doi.org/1 $0.1159 / 000096043$

64. Brandys MK, Slof-Op't Landt MC, van Elburg AA et al (2012) Anorexia nervosa and the Val158Met polymorphism of the COMT gene: meta-analysis and new data. Psychiatr Genet 22(3):130-136. https://doi.org/10.1097/YPG. 0b013e328351859e

65. Yilmaz Z, Kaplan AS, Tiwari AK, Levitan RD, Piran S, Bergen AW, Kaye WH, Hakonarson H, Wang K, Berrettini WH, Brandt HA, Bulik CM, Crawford S, Crow S, Fichter MM, Halmi KA, Johnson CL, Keel PK, Klump KL, Magistretti P, Mitchell JE, Strober M, Thornton LM, Treasure J, Woodside DB, Knight J, Kennedy JL (2014) The role of leptin, melanocortin, and neurotrophin system genes on body weight in anorexia nervosa and bulimia nervosa. J Psychiatr Res 55:77-86. https://doi.org/10.1016/j.jpsychires.2014.04.005

66. Quinton ND, Meechan DW, Brown K, Eastwood H, Blakemore AIF (2004) Single nucleotide polymorphisms in the leptin receptor gene: studies in anorexia nervosa. Psychiatr Genet 14(4):191-194. https://doi.org/10.1097/ 00041444-200412000-00004

67. Dardennes RM, Zizzari P, Tolle V, Foulon C, Kipman A, Romo L, lancuGontard D, Boni C, Sinet PM, Thérèse Bluet M, Estour B, Mouren MC, Guelfi $J$ D, Rouillon F, Gorwood P, Epelbaum J (2007) Family trios analysis of common polymorphisms in the obestatin/ghrelin, BDNF and AGRP genes in patients with Anorexia nervosa: association with subtype, body-mass index, severity and age of onset. Psychoneuroendocrinology 32(2):106-113. https://doi.org/10.1016/.jpsyneuen.2006.11.003

68. Cellini E, Nacmias B, Brecelj-Anderluh M, Badía-Casanovas A, Bellodi L, Boni C, di Bella D, Estivill X, Fernandez-Aranda F, Foulon C, Friedel S, Gabrovsek M, Gorwood P, Gratacos M, Guelfi J, Hebebrand J, Hinney A, Holliday J, Hu X, Karwautz A, Kipman A, Komel R, Rotella CM, Ribases M, Ricca V, Romo L, Tomori M, Treasure J, Wagner G, Collier DA, Sorbi S, EC Framework V 'Factors in Healthy Eating' consortium (2006) Case-control and combined family trios analysis of three polymorphisms in the ghrelin gene in European patients with anorexia and bulimia nervosa. Psychiatr Genet 16(2): 51-52. https://doi.org/10.1097/01.ypg.0000194444.89436.e9

69. Ando T, Komaki G, Naruo T, Okabe K, Takii M, Kawai K, Konjiki F, Takei M, Oka T, Takeuchi K, Masuda A, Ozaki N, Suematsu H, Denda K, Kurokawa N, Itakura K, Yamaguchi C, Kono M, Suzuki T, Nakai Y, Nishizono-Maher A, Koide M, Murakami K, Nagamine K, Tomita Y, Ookuma K, Tomita K, Tonai E, Ooshima A, Ishikawa T, Ichimaru Y (2006) Possible role of preproghrelin gene polymorphisms in susceptibility to bulimia nervosa. Am J Med Genetics Part B Neuropsychiatric Genetics 141B(8):929-934. https://doi.org/1 0.1002/ajmg.b.30387

70. Branson R, Potoczna N, Kral JG, Lentes KU, Hoehe MR, Horber FF (2003) Binge eating as a major phenotype of melanocortin 4 receptor gene mutations. N Engl J Med 348(12):1096-1103. https://doi.org/10.1056/ NEJMoa021971

71. Hebebrand J, Geller F, Dempfle A, Heinzel-Gutenbrunner M, Raab M, Gerber G, Wermter AK, Horro FF, Blundell J, Schäfer H, Remschmidt H, Herpertz S,
Hinney A (2004) Binge-eating episodes are not characteristic of carriers of melanocortin-4 receptor gene mutations. Mol Psychiatry 9(8):796-800. https://doi.org/10.1038/sj.mp.4001491

72. Brandys MK, van Elburg AA, Loos RJF, Bauer F, Hendriks J, van der Schouw YT, Adan RAH (2010) Are recently identified genetic variants regulating BMI in the general population associated with anorexia nervosa? American journal of medical genetics Part B. Neuropsychiatric Genetics 153B(2):695699. https://doi.org/10.1002/ajmg.b.31026

73. Vink T, Hinney A, van Elburg AA, van Goozen SHM, Sandkuijl LA, Sinke RJ, Herpertz-Dahlmann BM, Hebebrand J, Remschmidt $H$, van Engeland $H$, Adan RAH (2001) Association between an agouti-related protein gene polymorphism and anorexia nervosa. Mol Psychiatry 6(3):325-328. https:// doi.org/10.1038/s.mp.4000854

74. Versini A, Ramoz N, Le Strat Y et al (2010) Estrogen receptor 1 gene (ESR1) is associated with restrictive anorexia nervosa. Neuropsychopharmacology 35(8):1818-1825. https://doi.org/10.1038/npp.2010.49

75. Eastwood H, Brown KM, Markovic D, Pieri LF (2002) Variation in the ESR1 and ESR2 genes and genetic susceptibility to anorexia nervosa. Mol Psychiatry 7(1):86-89. https://doi.org/10.1038/sj/mp/4000929

76. Rosenkranz K, Hinney A, Ziegler A, Hermann H, Fichter M, Mayer H, Siegfried W, Young JK, Remschmidt H, Hebebrand J (1998) Systematic mutation screening of the estrogen receptor beta gene in probands of different weight extremes: identification of several genetic variants. J Clin Endocrinol Metab 83(12):4524-4527. https://doi.org/10.1210/jcem. 83.12.5471

77. Nilsson M, Naessen S, Dahlman I et al (2004) Association of estrogen receptor beta gene polymorphisms with bulimic disease in women. Mol Psychiatry 9(1):28-34. https://doi.org/10.1038/s.mp.4001402

78. Ribases M, Gratacos M, Armengol L et al (2003) Met66 in the brainderived neurotrophic factor (BDNF) precursor is associated with anorexia nervosa restrictive type. Mol Psychiatry 8(8):745-751. https:// doi.org/10.1038/sj.mp.4001281

79. Ribases M, Gratacos M, Fernandez-Aranda F et al (2004) Association of BDNF with anorexia, bulimia and age of onset of weight loss in six European populations. Hum Mol Genet 13(12):1205-1212. https://doi.org/10.1093/ $\mathrm{hmg} / \mathrm{ddh} 137$

80. Muller TD, Reichwald K, Bronner G et al (2008) Lack of association of genetic variants in genes of the endocannabinoid system with anorexia nervosa. Child Adolesc Psychiatry Ment Health 2(1):33. https://doi.org/10.1186/1753-2 000-2-33

81. Monteleone P, Bifulco M, Di Filippo C et al (2009) Association of CNR1 and FAAH endocannabinoid gene polymorphisms with anorexia nervosa and bulimia nervosa: evidence for synergistic effects. Genes Brain Behav 8(7): 728-732. https://doi.org/10.1111/j.1601-183X.2009.00518.X

82. Davis CA, Levitan RD, Reid C, Carter JC, Kaplan AS, Patte KA, King N, Curtis C, Kennedy JL (2009) Dopamine for "wanting" and opioids for "liking": a comparison of obese adults with and without binge eating. Obesity 17(6): 1220-1225. https://doi.org/10.1038/oby.2009.52

83. Muller TD, Greene BH, Bellodi L et al (2012) Fat mass and obesity-associated gene (FTO) in eating disorders: evidence for association of the rs9939609 obesity risk allele with bulimia nervosa and anorexia nervosa. Obes Facts 5(3):408-419. https://doi.org/10.1159/000340057

84. Jonassaint CR, Szatkiewicz JP, Bulik CM, Thornton LM, Bloss C, Berrettini WH, Kaye WH, Bergen AW, Magistretti P, Strober M, Keel PK, Brandt H, Crawford S, Crow S, Fichter MM, Goldman D, Halmi KA, Johnson C, Kaplan AS, Klump KL, la Via M, Mitchell JE, Rotondo A, Treasure J, Woodside DB (2011) Absence of association between specific common variants of the obesityrelated FTO gene and psychological and behavioral eating disorder phenotypes. Am J Med Genetics Part B Neuropsychiatric Genetics 156B(4): 454-461. https://doi.org/10.1002/ajmg.b.31182

85. Slof-Op't Landt MC, Meulenbelt I, Bartels M et al (2011) Association study in eating disorders: TPH2 associates with anorexia nervosa and self-induced vomiting. Genes Brain Behav 10(2):236-243. https://doi.org/10.1111/j.1601-1 83X.2010.00660.X

86. Hinney A, Becker I, Heibult O et al (1998) Systematic mutation screening of the pro-opiomelanocortin gene: identification of several genetic variants including three different insertions, one nonsense and two missense point mutations in probands of different weight extremes. J Clin Endocrinol Metab 83(10):3737-3741. https://doi.org/10.1210/jcem.83.10.5298

87. Mercader JM, Saus E, Aguera Z et al (2008) Association of NTRK3 and its interaction with NGF suggest an altered cross-regulation of the 
neurotrophin signaling pathway in eating disorders. Hum Mol Genet 17(9): 1234-1244. https://doi.org/10.1093/hmg/ddn013

88. Duncan L, Yilmaz Z, Gaspar H, Walters R, Goldstein J, Anttila V, Bulik-Sullivan B, Ripke S, Eating Disorders Working Group of the Psychiatric Genomics Consortium, Thornton L, Hinney A, Daly M, Sullivan PF, Zeggini E, Breen G, Bulik CM, Duncan L, Yilmaz Z, Gaspar H, Walters R, Goldstein J, Anttila V, BulikSullivan B, Ripke S, Adan R, Alfredsson L, Ando T, Andreassen O, Aschauer H, Baker J, Barrett J, Bencko V, Bergen A, Berrettini W, Birgegård A, Boni C, Perica VB, Brandt H, Burghardt R, Carlberg L, Cassina M, Cesta C, Cichon S, Clementi M, Cohen-Woods S, Coleman J, Cone R, Courtet P, Crawford S, Crow S, Crowley J, Danner U, Davis O, de Zwaan M, Dedoussis G, Degortes D, DeSocio J, Dick D, Dikeos D, Dina C, Ding B, Dmitrzak-Weglarz M, Docampo E, Egberts K, Ehrlich S, Escaramís G, Esko T, Espeseth T, Estivill X, Favaro A, FernándezAranda F, Fichter M, Finan C, Fischer K, Floyd J, Föcker M, Foretova L, Forzan M, Fox C, Franklin C, Gaborieau V, Gallinger S, Gambaro G, Giegling I, Gonidakis F, Gorwood P, Gratacos M, Guillaume S, Guo Y, Hakonarson H, Halmi K, Harrison R, Hatzikotoulas K, Hauser J, Hebebrand J, Helder S, Hendriks J, Herms S, Herpertz-Dahlmann B, Herzog W, Hilliard C, Huckins L, Hudson J, Huemer J, Imgart $H$, Inoko $H$, Jall $S$, Jamain $S$, Janout $V$, Jiménez-Murcia $S$, Johnson $C$, Jordan J, Julià A, Juréus A, Kalsi G, Kaplan A, Kaprio J, Karhunen L, Karwautz A, Kas M, Kaye W, Kennedy M, Kennedy J, Keski-Rahkonen A, Kiezebrink K, Kim YR, Klareskog L, Klump K, Knudsen GP, Koeleman B, Koubek D, la Via M, Landén M, le Hellard S, Leboyer M, Levitan R, Li D, Lichtenstein P, Lilenfeld L, Lissowska J, Lundervold A, Magistretti P, Maj M, Mannik K, Marsal S, Kaminska D, Martin N, Mattingsdal M, McDevitt S, McGuffin P, Merl E, Metspalu A, Meulenbelt I, Micali N, Mitchell J, Mitchell K, Monteleone P, Monteleone AM, Montgomery G, Mortensen P, Munn-Chernoff M, Müller T, Nacmias B, Navratilova M, Nilsson I, Norring C, Ntalla I, Ophoff R, O'Toole J, Palotie A, Pantel J, Papezova H, Parker R, Pinto D, Rabionet R, Raevuori A, Rajewski A, Ramoz N, Rayner NW, ReichbornKjennerud T, Ricca V, Ripatti S, Ritschel F, Roberts M, Rotondo A, Rujescu D, Rybakowski F, Santonastaso P, Scherag A, Scherer S, Schmidt U, Schork N, Schosser A, Scott L, Seitz J, Slachtova L, Sladek R, Slagboom PE, 't Landt MSO, Slopien A, Smith T, Soranzo N, Sorbi S, Southam L, Steen V, Strengman E, Strober M, Szatkiewicz J, Szeszenia-Dabrowska N, Tachmazidou I, Tenconi E, Tortorella A, Tozzi F, Treasure J, Tschöp M, Tsitsika A, Tziouvas K, van Elburg A, van Furth E, Wade T, Wagner G, Walton E, Watson H, Wichmann HE, Widen E, Woodside DB, Yanovski J, Yao S, Zerwas S, Zipfel S, Thornton L, Hinney A, Daly M, Sullivan PF, Zeggini E, Breen G, Bulik CM (2017) Significant locus and metabolic genetic correlations revealed in genome-wide association study of anorexia nervosa. Am J Psychiatry 174(9):850-858. https://doi.org/10.1176/a ppi.ajp.2017.16121402

89. Nordsletten AE, Larsson H, Crowley JJ, Almqvist C, Lichtenstein P, MataixCols D (2016) Patterns of Nonrandom Mating Within and Across 11 Major Psychiatric Disorders. JAMA Psychiatry 73(4):354-361. https://doi.org/10.1 001/jamapsychiatry.2015.3192

90. Dina C, Meyre D, Gallina S, Durand E, Korner A, Jacobson P, Carlsson LM, Kiess W, Vatin V, Lecoeur C, Delplanque J et al (2007) Variation in FTO contributes to childhood obesity and severe adult obesity. Nat Genet 39(6): 724-726. https://doi.org/10.1038/ng2048

91. Thorleifsson G, Walters GB, Gudbjartsson DF, Steinthorsdottir V, Sulem P, Helgadottir A, Styrkarsdottir U, Gretarsdottir S, Thorlacius S, Jonsdottir I, Jonsdottir T, Olafsdottir EJ, Olafsdottir GH, Jonsson T, Jonsson F, BorchJohnsen K, Hansen T, Andersen G, Jorgensen T, Lauritzen T, Aben KK, Verbeek ALM, Roeleveld N, Kampman E, Yanek LR, Becker LC, Tryggvadottir L, Rafnar T, Becker DM, Gulcher J, Kiemeney LA, Pedersen O, Kong A, Thorsteinsdottir U, Stefansson K (2009) Genome-wide association yields new sequence variants at seven loci that associate with measures of obesity. Nat Genet 41(1):18-24. https://doi.org/10.1038/ng.274

92. Srisai D, Gillum MP, Panaro BL, Zhang XM, Kotchabhakdi N, Shulman Gl, Ellacott KL, Cone RD (2011) Characterization of the hyperphagic response to dietary fat in the MC4R knockout mouse. Endocrinology 152(3):890-902. https://doi.org/10.1210/en.2010-0716

93. Yeo GS, Farooqi IS, Aminian S et al (1998) A frameshift mutation in MC4R associated with dominantly inherited human obesity. Nat Genet 20(2):111112. https://doi.org/10.1038/2404

94. Benzinou M, Creemers JW, Choquet $\mathrm{H}$ et al (2008) Common nonsynonymous variants in PCSK1 confer risk of obesity. Nat Genet 40(8): 943-945. https://doi.org/10.1038/ng.177

95. Liu YJ, Liu XG, Wang L, Dina C, Yan H, Liu JF, Levy S, Papasian CJ, Drees BM, Hamilton JJ, Meyre D, Delplanque J, Pei YF, Zhang L, Recker RR, Froguel P, Deng HW (2008) Genome-wide association scans identified CTNNBL1 as a novel gene for obesity. Hum Mol Genet 17(12):1803-1813. https://doi.org/1 0.1093/hmg/ddn072

96. Scherag A, Dina C, Hinney A, Vatin V, Scherag S, Vogel CIG, Müller TD, Grallert H, Wichmann HE, Balkau B, Heude B, Jarvelin MR, Hartikainen AL, Levy-Marchal C, Weill J, Delplanque J, Körner A, Kiess W, Kovacs P, Rayner NW, Prokopenko I, McCarthy MI, Schäfer $H$, Jarick I, Boeing H, Fisher E, Reinehr T, Heinrich J, Rzehak P, Berdel D, Borte M, Biebermann H, Krude H, Rosskopf D, Rimmbach C, Rief W, Fromme T, Klingenspor M, Schürmann A, Schulz N, Nöthen MM, Mühleisen TW, Erbel R, Jöckel KH, Moebus S, Boes T, Illig T, Froguel P, Hebebrand J, Meyre D (2010) Two new Loci for bodyweight regulation identified in a joint analysis of genome-wide association studies for early-onset extreme obesity in French and german study groups. PLoS Genet 6(4):e1000916. https://doi.org/10.1371/journal.pgen.1000916

97. Willer CJ, Speliotes EK, Loos RJ, Li S, Lindgren CM, Heid IM, Berndt SI, Elliott AL, Jackson AU, Lamina C, Lettre G, Lim N, Lyon HN, McCarroll S, Papadakis K, Qi L, Randall JC, Roccasecca RM, Sanna S, Scheet P, Weedon MN, Wheeler E, Zhao JH, Jacobs LC, Prokopenko I, Soranzo N, Tanaka T, Timpson NJ, Almgren P, Bennett A, Bergman RN, Bingham SA, Bonnycastle LL, Brown M, Burtt NP, Chines P, Coin L, Collins FS, Connell JM, Cooper C, Smith GD, Dennison EM, Deodhar P, Elliott P, Erdos MR, Estrada K, Evans DM, Gianniny L, Gieger C, Gillson CJ, Guiducci C, Hackett R, Hadley D, Hall AS, Havulinna AS, Hebebrand J, Hofman A, Isomaa B, Jacobs KB, Johnson T, Jousilahti P, Jovanovic Z, Khaw KT, Kraft P, Kuokkanen M, Kuusisto J, Laitinen J, Lakatta EG, Luan J, Luben RN, Mangino M, McArdle W, Meitinger T, Mulas A, Munroe PB, Narisu N, Ness AR, Northstone K, O'Rahilly S, Purmann C, Rees MG, Ridderstråle M, Ring SM, Rivadeneira F, Ruokonen A, Sandhu MS, Saramies J, Scott L, Scuteri A, Silander K, Sims MA, Song K, Stephens J, Stevens $S$, Stringham HM, Tung YC, Valle TT, van Duijn C, Vimaleswaran KS, Vollenweider P, Waeber G, Wallace C, Watanabe RM, Waterworth DM, Watkins N, Wellcome Trust Case Control Consortium, Witteman JC, Zeggini E, Zhai G, Zillikens MC, Altshuler D, Caulfield MJ, Chanock SJ, Farooqi IS, Ferrucci L, Guralnik JM, Hattersley AT, Hu FB, Jarvelin MR, Laakso M, Mooser V, Ong KK, Ouwehand WH, Salomaa V, Samani NJ, Spector TD, Tuomi T, Tuomilehto J, Uda M, Uitterlinden AG, Wareham NJ, Deloukas P, Frayling TM, Groop LC, Hayes RB, Hunter DJ, Mohlke KL, Peltonen L, Schlessinger D, Strachan DP, Wichmann HE, McCarthy M, Boehnke M, Barroso I, Abecasis GR, Hirschhorn JN, Genetic Investigation of ANthropometric Traits Consortium (2009) Six new loci associated with body mass index highlight a neuronal influence on body weight regulation. Nat Genet 41(1):25-34. https://doi.org/10.1038/ng.287

98. Berndt SI, Gustafsson S, Magi R et al (2013) Genome-wide meta-analysis identifies 11 new loci for anthropometric traits and provides insights into genetic architecture. Nat Genet 45(5):501-512. https://doi.org/10.1 038/ng.2606

99. Meyre D, Delplanque J, Chevre JC et al (2009) Genome-wide association study for early-onset and morbid adult obesity identifies three new risk loci in European populations. Nat Genet 41(2):157-159. https://doi.org/10.1038/ng.301

100. Lindgren CM, Heid IM, Randall JC, Lamina C, Steinthorsdottir V, Qi L, Speliotes EK, Thorleifsson G, Willer CJ, Herrera BM, Jackson AU, Lim N, Scheet P, Soranzo N, Amin N, Aulchenko YS, Chambers JC, Drong A, Luan J, Lyon HN, Rivadeneira F, Sanna S, Timpson NJ, Zillikens MC, Zhao JH, Almgren P, Bandinelli S, Bennett AJ, Bergman RN, Bonnycastle LL, Bumpstead SJ, Chanock SJ, Cherkas L, Chines P, Coin L, Cooper C, Crawford G, Doering A, Dominiczak A, Doney AS, Ebrahim S, Elliott P, Erdos MR, Estrada K, Ferrucci L, Fischer G, Forouhi NG, Gieger C, Grallert H, Groves CJ, Grundy S, Guiducci C, Hadley D, Hamsten A, Havulinna AS, Hofman A, Holle R, Holloway JW, Illig T, Isomaa B, Jacobs LC, Jameson K, Jousilahti P, Karpe F, Kuusisto J, Laitinen J, Lathrop GM, Lawlor DA, Mangino M, McArdle W, Meitinger T, Morken MA, Morris AP, Munroe P, Narisu N, Nordström A, Nordström P, Oostra BA, Palmer CN, Payne F, Peden JF, Prokopenko I, Renström F, Ruokonen A, Salomaa V, Sandhu MS, Scott LJ, Scuteri A, Silander K, Song K, Yuan X, Stringham HM, Swift AJ, Tuomi T, Uda M, Vollenweider P, Waeber G, Wallace C, Walters GB, Weedon MN, Wellcome Trust Case Control Consortium, Witteman JC, Zhang C, Zhang W, Caulfield MJ, Collins FS, Davey Smith G, Day IN, Franks PW, Hattersley AT, Hu FB, Jarvelin MR, Kong A, Kooner JS, Laakso M, Lakatta E, Mooser V, Morris AD, Peltonen L, Samani NJ, Spector TD, Strachan DP, Tanaka T, Tuomilehto J, Uitterlinden AG, van Duijn C, Wareham NJ, Hugh Watkins, Procardis Consortia, Waterworth DM, Boehnke M, Deloukas P, Groop L, Hunter DJ, Thorsteinsdottir U, Schlessinger D, Wichmann HE, Frayling TM, Abecasis GR, 
Hirschhorn JN, Loos RJ, Stefansson K, Mohlke KL, Barroso I, McCarthy M, Giant Consortium (2009) Genome-wide association scan meta-analysis identifies three Loci influencing adiposity and fat distribution. PLoS Genet 5(6):e1000508. https://doi.org/10.1371/journal.pgen.1000508

101. Heard-Costa NL, Zillikens MC, Monda KL, Johansson $\AA$, Harris TB, Fu M, Haritunians T, Feitosa MF, Aspelund T, Eiriksdottir G, Garcia M, Launer LJ, Smith AV, Mitchell BD, McArdle PF, Shuldiner AR, Bielinski SJ, Boerwinkle E, Brancati F, Demerath EW, Pankow JS, Arnold AM, Chen YDI, Glazer NL, McKnight B, Psaty BM, Rotter JI, Amin N, Campbell H, Gyllensten U, Pattaro C, Pramstaller PP, Rudan I, Struchalin M, Vitart V, Gao X, Kraja A, Province MA, Zhang Q, Atwood LD, Dupuis J, Hirschhorn JN, Jaquish CE, O'Donnell CJ, Vasan RS, White CC, Aulchenko YS, Estrada K, Hofman A, Rivadeneira F, Uitterlinden AG, Witteman JCM, Oostra BA, Kaplan RC, Gudnason V, O'Connell JR, Borecki IB, van Duijn CM, Cupples LA, Fox CS, North KE (2009) NRXN3 is a novel locus for waist circumference: a genome-wide association study from the CHARGE Consortium. PLoS Genet 5(6):e1000539. https://doi. org/10.1371/journal.pgen.1000539

102. Cho YS, Go MJ, Kim YJ, Heo JY, Oh JH, Ban HJ, Yoon D, Lee MH, Kim DJ, Park M, Cha SH, Kim JW, Han BG, Min H, Ahn Y, Park MS, Han HR, Jang HY, Cho EY, Lee JE, Cho NH, Shin C, Park T, Park JW, Lee JK, Cardon L, Clarke G, McCarthy MI, Lee JY, Lee JK, Oh B, Kim HL (2009) A large-scale genomewide association study of Asian populations uncovers genetic factors influencing eight quantitative traits. Nat Genet 41(5):527-534. https://doi. org/10.1038/ng.357

103. Speliotes EK, Willer CJ, Berndt SI et al (2010) Association analyses of 249,796 individuals reveal 18 new loci associated with body mass index. Nat Genet 42(11):937-948. https://doi.org/10.1038/ng.686

104. Heid IM, Jackson AU, Randall JC et al (2010) Meta-analysis identifies 13 new loci associated with waist-hip ratio and reveals sexual dimorphism in the genetic basis of fat distribution. Nat Genet 42(11):949-960. https://doi.org/1 0.1038/ng.685

105. Locke AE, Kahali B, Berndt SI et al (2015) Genetic studies of body mass index yield new insights for obesity biology. Nature 518(7538):197-206. https://doi.org/10.1038/nature14177

106. Yazdi FT, Clee SM, Meyre D (2015) Obesity genetics in mouse and human: back and forth, and back again. PeerJ 3:e856. https://doi.org/10. 7717/peerj.856

107. Ichimura A, Hirasawa A, Poulain-Godefroy O, Bonnefond A, Hara T, Yengo L, Kimura I, Leloire A, Liu N, lida K, Choquet H, Besnard P, Lecoeur C, Vivequin S, Ayukawa K, Takeuchi M, Ozawa K, Tauber M, Maffeis C, Morandi A, Buzzetti R, Elliott P, Pouta A, Jarvelin MR, Körner A, Kiess W, Pigeyre M, Caiazzo R, van Hul W, van Gaal L, Horber F, Balkau B, Lévy-Marchal C, Rouskas K, Kouvatsi A, Hebebrand J, Hinney A, Scherag A, Pattou F, Meyre D, Koshimizu TA, Wolowczuk I, Tsujimoto G, Froguel P (2012) Dysfunction of lipid sensor GPR120 leads to obesity in both mouse and human. Nature 483(7389):350-354. https://doi.org/10.1038/nature10798

108. Jiao H, Arner P, Hoffstedt J, Brodin D, Dubern B, Czernichow S, van't Hooft F, Axelsson T, Pedersen O, Hansen T, Sørensen TIA, Hebebrand J, Kere J, Dahlman-Wright K, Hamsten A, Clement K, Dahlman I (2011) Genome wide association study identifies KCNMA1 contributing to human obesity. BMC Med Genet 4(1):51. https://doi.org/10.1186/1755-8794-4-51

109. Okada Y, Kubo M, Ohmiya $\mathrm{H}$ et al (2012) Common variants at CDKAL1 and KLF9 are associated with body mass index in east Asian populations. Nat Genet 44(3):302-306. https://doi.org/10.1038/ng.1086

110. Wheeler E, Huang N, Bochukova EG, Keogh JM, Lindsay S, Garg S, Henning E, Blackburn H, Loos RJF, Wareham NJ, O'Rahilly S, Hurles ME, Barroso I, Farooqi IS (2013) Genome-wide SNP and CNV analysis identifies common and low-frequency variants associated with severe early-onset obesity. Nat Genet 45(5):513-517. https://doi.org/10.1038/ng.2607

111. Scuteri A, Sanna S, Chen WM, Uda M, Albai G, Strait J, Najiar S, Nagaraja R, Orrú M, Usala G, Dei M, Lai S, Maschio A, Busonero F, Mulas A, Ehret GB, Fink AA, Weder AB, Cooper RS, Galan P, Chakravarti A, Schlessinger D, Cao A, Lakatta E, Abecasis GR (2007) Genome-wide association scan shows genetic variants in the FTO gene are associated with obesity-related traits. PLoS Genet 3(7):e115. https://doi.org/10.1371/journal.pgen.0030115

112. Heatherton TF, Polivy J, Herman CP (1991) Restraint, weight loss, and variability of body weight. J Abnorm Psychol 100(1):78-83. https://doi.org/1 $0.1037 / / 0021-843 \times 100.1 .78$

113. Stice $E$ (2002) Risk and maintenance factors for eating pathology: a metaanalytic review. Psychol Bull 128(5):825-848. https://doi.org/10.1037/0033-2 909.128.5.825
114. Hill AJ (2004) Does dieting make you fat? Br J Nutr 92(Suppl 1):S15-S18. https://doi.org/10.1079/bjn20041135

115. Field AE, Austin SB, Taylor CB, Malspeis S, Rosner B, Rockett HR, Gillman MW, Colditz GA (2003) Relation between dieting and weight change among preadolescents and adolescents. Pediatrics 112(4):900-906. https://doi.org/1 0.1542/peds.112.4.900

116. Stice E, Presnell K, Shaw H, Rohde P (2005) Psychological and behavioral risk factors for obesity onset in adolescent girls: a prospective study. J Consult Clin Psychol 73(2):195-202. https://doi.org/10.1037/0022-006X.73.2.195

117. Butryn ML, Wadden TA (2005) Treatment of overweight in children and adolescents: does dieting increase the risk of eating disorders? Int J Eat Disord 37(4):285-293. https://doi.org/10.1002/eat.20098

118. Walker LL, Gately PJ, Bewick BM, Hill AJ (2003) Children's weight-loss camps: psychological benefit or jeopardy? Int J Obesity RelatMetab Disord 27(6): 748-754. https://doi.org/10.1038/sj.ijo.0802290

119. Neumark-Sztainer D (2005) Can we simultaneously work toward the prevention of obesity and eating disorders in children and adolescents? Int J Eat Disord 38(3):220-227. https://doi.org/10.1002/eat.20181

120. O'Dea JA (2005) Prevention of child obesity: 'first, do no harm'. Health Educ Res 20(2):259-265. https://doi.org/10.1093/her/cyg116

\section{Publisher's Note}

Springer Nature remains neutral with regard to jurisdictional claims in published maps and institutional affiliations.

\section{Submit your manuscript to a SpringerOpen ${ }^{\circ}$ journal and benefit from:}

- Convenient online submission

- Rigorous peer review

- Open access: articles freely available online

High visibility within the field

- Retaining the copyright to your article

Submit your next manuscript at $>$ springeropen.com 